\title{
An Adaptive Complex Data Investigation Methodology using Interactive Multi-Menus
}

\author{
Ioannis Deliyiannis ${ }^{1}$, Andreas Floros ${ }^{2}$ and Michael F. Webster ${ }^{3}$ \\ 1,2 Dept. of Audiovisual Arts, Ionian University, Corfu, Greece, \\ \{yiannis, floros@ionio.gr \\ ${ }^{3}$ Institute of Non-Newtonian Fluid Mechanics, University of Wales Swansea, \\ Computer Science Department, Singleton Park, SA2 8PP, Wales, UK, \\ \{m.f.webster@swan.ac.uk\}
}

\begin{abstract}
Multimedia presentation of dynamic rheological experimental and simulated data sets is a complex process, due to the dynamic interrelation of the data. In this work we address the problem of direct data interaction, focusing particularly on the case where the application has to dynamically handle changes at runtime. Interactivity modeling relies on visual metadata representations, which are both used to relate directly to the underlying system-graph and formally describe content-connectivity. Under this context a novel user-interaction structure (Multi-Menu) is introduced, allowing valid-only data comparison across the vast number of possible results combinations, supporting discussion-based data combinations and user-driven scenarios, which can in turn trigger automated simulation execution to generate missing data. The proposed methodology applies to other complex data investigation scenarios and systems with adaptive interactivity and automatic system-adjustment requirements.
\end{abstract}

Keywords: adaptive investigation, Multi-Menus, framework, model, metadata

\section{Introduction}

This work introduces a user interface construction methodology used for interactive multimedia investigation of scientific and rheological data. The multimedia content used under the case-study is the end-result of simulation or experimental studies and is provided in visualised post-processed form. Under the current case study, results are generated for model fluids in a two-dimensional (2D) setting. Further investigations included 3D and free-surface modeling, validated against results obtained from actual kneading experiments. Fully and part-filled cases were considered, in vertical and horizontal mixer orientations. The main objectives of this work is to demonstrate the navigation flexibility through the vast content-domain of the selected case study, under various presentation modes and to identify the principal factors, which lead to the reduction of time and cost of mix- 
ing by appropriate adjustment of stirrer design. This is achieved through extensive data visualisation, supported by a graphical interaction mechanism. Added complication is introduced as data are calculated and delivered by multiple research groups, working in parallel, particularly as each group employs individual methods to represent the data. Experimental and simulation results generate a variety of investigation areas, introducing a large number of variables.

Visual metadata are employed to build the human-computer interaction subsystem, enabling complete, direct and meaningful presentation of the associated data. This is achieved by creating an XML-based multimedia visualisation database and a controlling interface, supporting interactive user-driven interrogation and illustration scenarios of the key factors that affect the process of dough kneading. Combinations of novel interaction techniques are realised, together with predetermined modes of interaction (cruise-control/data-navigation). The current industrially-related case study combines a non-linear access mechanism, where system attributes (such as pre-defined cruise-control paths and hierarchical presentation orders) may be dynamically multiplexed, enabling various abstract datainvestigation levels to be activated. The sheer size of data introduces storage/retrieval limitations, particularly as non-linear access is supported by the endsystem. Synchronous display of multiple streams results in system/networkbottlenecks, particularly as delivery over Internet communication channels is required. In addition, presentation of the data in a meaningful manner has triggered the need for advanced and multiplexed navigational routes, which permit various investigative scenarios, alongside pre-determined presentation-paths. In Fig. 1 for example, various animated data are presented simultaneously, while the system permits either the selection of a deterministic presentation path, or direct interaction via option-selection of the multi-menu icons displayed on the left.

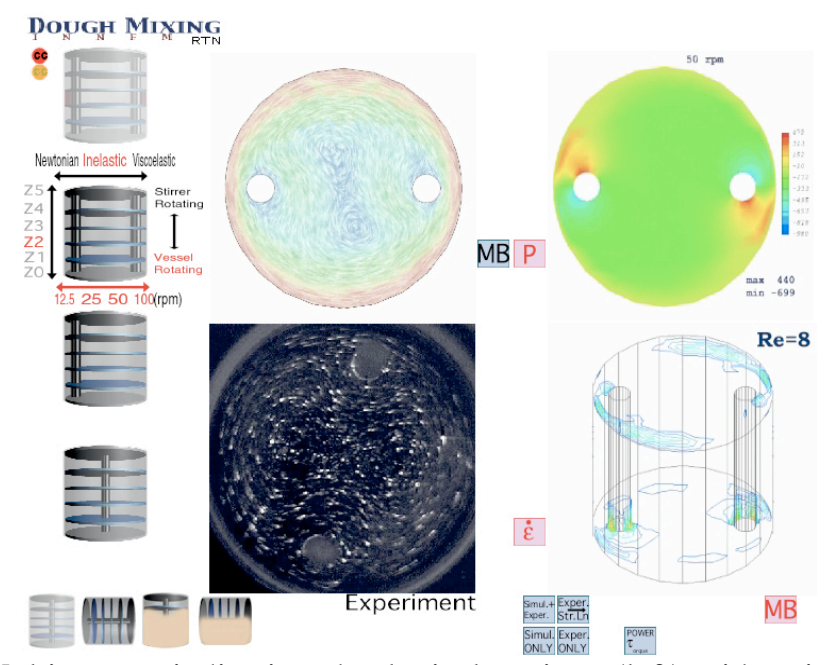

Fig. 1. Multi-menus indicating rheological settings (left) with animated motionblur, pressure, laser-scatter and three-dimensional extension data, for an inelastic fluid with mixing speeds between 12.5 to $100 \mathrm{rpm}, \mathrm{Z} 2$ plane, rotating vessel. 
MMS data integration ([9]), [15]) has enabled effortless navigation through vast volume of information, dispensation of data-duplication, and interactive comparison of experimental and simulation animations, within a single MMS frame. A frame may be described as a holder for meta-data information, enabling multimedia data-instances to be grouped, categorised, synchronised, positioned, indexed, cached and displayed on-demand. For example, when two animations are to be displayed simultaneously, the interaction mechanism reads the metadata stored in both frames and subsequently adjusts appropriately the frame-rate, to display the data in synchronisation. In Fig. 1, four data frames are resized, synchronised and positioned automatically for the user to contrast fluid characteristics in both experimental and simulated settings. In addition, the MMS may be programmed to simulate common interaction modes [10].

The visualised content consists of experimental, 3D simulated and transient rheological data. This is accessed through parameter-adjustment and customisedmenus, reflecting design-choice and parameter-setting. Interface-design is based on the mixing-vessel design/orientation/fill and process parameters, as shown in Fig. 2. Comparison against experimentation introduces greater contentcomplexity. User-defined data-organisation, presentation and interactionparadigms are combined, to cover multiple levels of presentation-requirements. New interface styles for MMS data-presentation have been developed, using an hierarchical organisation, based on model adjustment and navigational graphs, introducing intelligent navigation [3], [16].

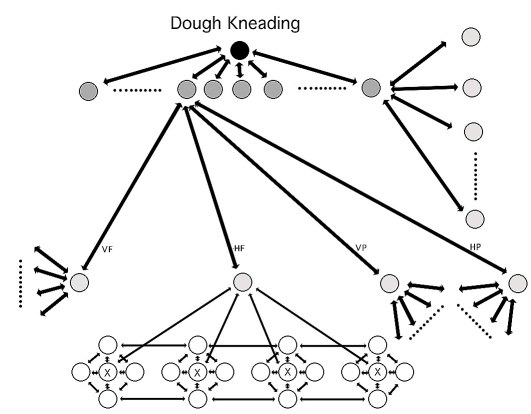

Fig. 2. Representation of the wide variety of research modes. $V$ indicates vertical mixer orientation used for bread mixing, $\mathrm{H}$ horizontal for biscuits, $\mathrm{P}$ for part-filled and $\mathrm{F}$ for fully-filled container.

Under the current case study, results are generated for Horizontal or Vertical Mixer Orientation; Incremental Stirrer Complexity for One Stirrer Concentric, One Stirrer Eccentric, Two Stirrers Eccentric (with/without) Baffles; Viscous and Viscoelastic Fluid-types; Speed of Kneading of 12.5, 25, 50 and 100 rotations per minute; 3D Investigation of the flow across depth for multiple planes; Fully and part-filled mixing; Free-surface in two and three dimensions, including transient development of the free surface; Stirrer shapes that include Full-Stirrer, HalfStirrer Horizontal, Half-Stirrer Vertical. Experimental fluids cover: model-fluids (for transparency), model-doughs (for material time-independency), to actual in- 
dustrial doughs. Each of the above considerations consists of various geometrical and parameter adjustments of incremental complexity. Fluid model representation ranges from Newtonian to inelastic, and finally, to viscoelastic. Adjustment of geometry and modelling complexity transcends from one stirrer, concentric to the final case of two stirrers, eccentric, free surface. Incrementation through experimental studies is outlined.

Frequently, calculations may be repeated with algorithm adjustments. Thus, each time data are received, increased complexity is introduced as it is necessary to identify unique, fresh data to be categorised and inserted, appropriately. Categorisation, ordering and duplication problems arise with propriety software and new data. The above deficiencies are amplified, as their inability to impose specific content-quality criteria, such as colour-depth and resolution, may introduce further comparison and conversion complexities. Within MMS, new data are inserted, or replaced, straightforwardly to the system without-preferred order. Some initial practical problems to be tackled include the collection, categorisation, visualisation and presentation of simulation results, having established data-sharing practice. This minimises data conversion, and as a consequence, the visualisation workload. The plethora and diversity of data, demands MMS techniques, relating to efficient content-updates and direct data-insertion.

\section{Scientific Interactive Multimedia Framework and Model}

Previous research has introduced a combined multimedia organisation and presentation framework [5], where interaction is classified between developers, users and observers in an MMS production and use stages. An overlapping setdiagram representation describes the relationships between the various groups, while a model describes content-connectivity using dynamically adapted graphs [6]. Framework-areas may be colored, to indicate individual and overlapping party-roles. Arrows may indicate group-interaction across the system. Set unions (A, B , C , D) indicate higher-order interaction, say between groups in constructionmode for a single-MMS, or indeed, a super-MMS, as in Fig. 3, right. Such a system is an implied product of the developer's domain.

Various interaction instances may be considered covering a wide range of scenarios. The area-exclusive scenario, which implies only limited interaction between the groups, where there are clearly defined expertise-boundaries. Each group describes its' requirements according to its' individual constraints. A common agreement across groups specifies the resulting MMS. For each group this is achieved without a detailed understanding of the mechanics of other groups. This is a typical scenario when, for example, a Multimedia company is employed to design an MMS. Line-diagrams may be used to specify relationships between these clearly defined groups in detail. 

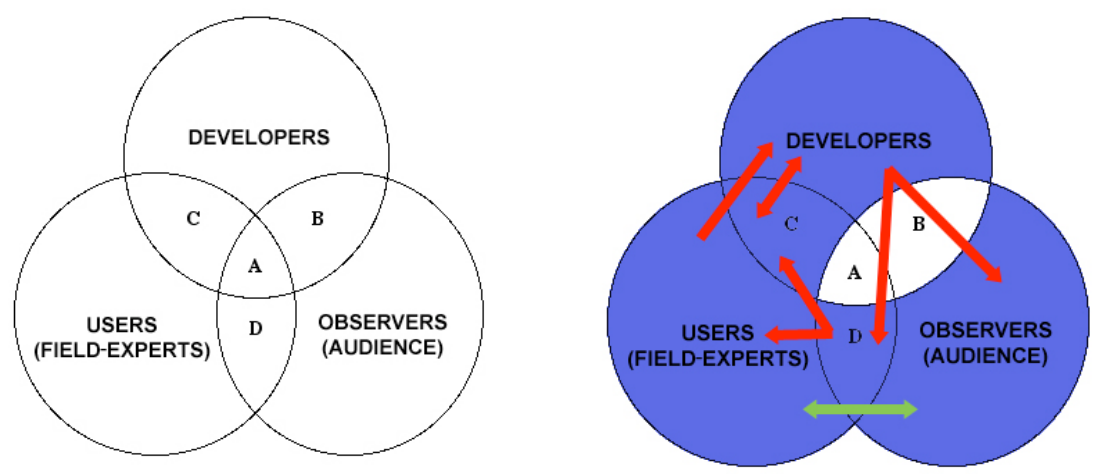

Fig. 3. The Scientific Interactive Multimedia Framework in various modes

A second scenario describes the instance where only "A" is colored. Here, the development group possesses knowledge that transcends all three groups. This would encompass the single-developer instance, where one has understanding of MM-development, expertise on the subject-matter and appreciates audience expectations. A third interaction scenario is identified when " $\mathrm{C}$ " and " $\mathrm{D}$ " only are highlighted. This setting implies that users/field-experts assume an active role in development (indicated by arrow), and commute between roles as user/audience. This accurately describes the development scenario of various scientific-level multimedia presentations. Bi-directional relationships may be indicated by doublearrows. In this manner, interaction between the parties involved may be described in a highly informative manner. The fourth instance describes a complex setting [Fig. 3, right], combining users (field-experts), developers and various audiencetypes (both versed and unversed in the subject matter). Here, the proposed framework achieves a higher-order description of the relationship between the parties involved. Varying levels of expertise may be described of increased complexity on-demand: users/field-experts (aware of development constraints), developers (aware of scientific context) and their interrelations. In such respect, traditional frameworks have been found lacking. By design, they were never intended to describe diverse configurations, in terms of expertise, content and development for an MMS. Instead, they assign further workload to a single party (usually the developer) to evaluate the quality of information. To interpret J. Carter [2]'...developers must understand the intended meaning of the data from the information providers, in order to evaluate its quality'. This is a particularly pertinent issue when considering research-content.

MM development relies on the Scientific Interactive Multimedia Model (SIMM), which addresses the issue of content / context connectivity through advanced representation means. The model transforms the system specification into an MMS by accommodating the multifaceted presentation requirements introduced with complex scientific-data [4]. Indexes encapsulating multimedia frames are used instead of individual media components and interrelationships between them. Each frame contains multiple media elements, such as text, sounds, images 
and meta-data, which are created automatically or may be inserted manually in XML format. Similar ideas have been cited in the literature, utilising a complex rule-based approach [8]. To emulate connectivity across lower-level content (such as single-streams), frames containing only a single media-element may be employed.

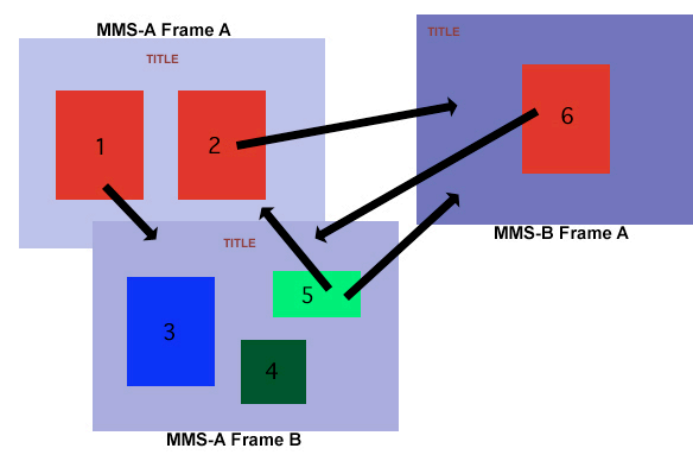

Fig. 4. Various linking scenarios for multimedia frames and streams

This approach poses multiple advantages, when compared to other cited in the literature [1]. First, low-level content connectivity is abstracted, as direct comparison is activated between two or more streams. In-turn, the volume of links is reduced. A second advantage is that individual media-components may be referenced separately, despite being organised within a more general structure. In addition, their ability to form further combinations is not reduced. This may be achieved separately, by being linked to other frames. Furthermore, a frame containing a title, and a set of streams provides immediate identification and categorisation of content. This introduces an advanced indexing mechanism, consisting of a frame-index, media-indexes (contained within each frame), and a set-of-states (in which a frame and a set of media are active). Content-linking is addressed using a "media-element to frame" referencing procedure. Each media-element may be employed as a user-interface construct. Fixed links may be programmed directly, or copied across other media-elements, reducing re-programming. Additionally, case-based links may be programmed locally (within frames of a singleMMS), unless external frame-access is necessary. Use of a knowledge-based approach (to dictate link-behaviour) is supported within the model via scripting.

Mathematical representation is used to formally describe the proposed model. From the computer science perspective, it is common to use mathematical constructs in system specification, automated verification and development [11]. Typical formal specification tools employ algebras, signatures and rules [13]. As a consequence, the functional representation chosen may be modeled directly through computer algorithms to enable automated presentation-construction and verification. 


\section{Multi-Menus, Graphs, Metadata and System Programming}

Multi-Menus are classified as a user-interface construction structure. These are linked directly to the underlying SIMM of an MMS. In this respect, visual media components such as icons, video-segments, complete video clips and text are used for frame-linking, which essentially is mapped to content-linking (see Fig. 4). When large content-domains are involved, complications are introduced, due to their unmanageable size and complexity in connectivity. This may result in multiple system-deficiencies, such as link- or interface-inconsistency. Therefore, linkmanagement protocols are introduced (at developmental stages), designed to allow consistent replication of frame-connectivity. This is achieved through the deployment of MM-templates, featuring fully functional external connectivity. When fresh data-content is appended, use of an appropriate template, with external links already earmarked, reduces programming to amending local-links.

Multi-Menus do not impose constraints in terms of the underlying development environment. The developer is allowed the flexibility to implement similar system-functionality, through a pick-and-mix approach. Hence, many non-MME technologies may be utilised. Examples include document-based construction tools (such as HTML pages utilising java applets), and hybrid combinations with other technologies, involving XML [12], ASP, JAVA, JSP, etc. The only requirement is dynamic-linking support. For example, plain HTML-pages cannot support Multi-Menus, since links point to a fixed address.

For MM-based user-interface development it is important to establish a detailed view of content interdependencies. The subsequent building step involves construction of user-interface structures. Here, these act as building blocks for userinterface/interaction. Having prepared all necessary media-components, one must develop the principal frames involved. In order to create a consistent userinterface for all sections, a single instance is developed by programming all the relative links once to all other relevant addresses. This reduces the programming effort as a copy of the complete interface is used as a template for new content. Current selection indication is achieved via setting particular icon-attributes to high-visibility. Visual icon-attributes such as low-visibility are also supported, where reduced visibility implies unavailable links. Having obtained a basis that is fully linked to the other instances, it remains to clone the completed frame. Copying or expanding the convoluted icons to share functionality across distinct frames achieves this. Adding the media-components themselves completes the task. Compared to the re-programming effort required when each multimedia instance is completed separately this is a speedy task. Also, it provides consistent iconplacement.

In addition, SIMM may be actively employed in the construction and functional extension of an MMS. In this manner, one aims to model content-connectivity [7] and interactivity. An additional user-interface structure is deemed necessary, in the construction of a front-end to underlying content. In that respect, "Multi-Menus", are employed to specify interface-component functionality, whilst relating to underlying content-frames. The principal reasons for using a Multimedia building- 
environment include: encapsulation of content, support for cross-platform delivery, system-extendibility and provision for fully functional scripting.

\section{Multi-Menus Development using Underlying Graph Structure}

Within the current case study, extensive use of graphs and related representation structures have been utilised for the rigorous implementation of interactive navigation. The type of graph used with this case study is a multi-connected graph [Fig. 2] For the organisation of simple slide-based presentations, a dynamic linked-list structure is employed. Each branch is itself a presentation, linked to external data (such as experimentally-captured and simulation-generated animations). This data categorisation is followed within the Multimedia organisation, but in a more liberal manner. Replacing strict-ordering of frames, data are retained locally to one instance in the MME. Links join the top-level to the case-study introduction, flyer-animation and project-plan slide-sorter. The second-level (indicated by dark-grey intensity) consists of the menus organised across geometry and setting-complexity (one-stirrer, concentric to two-stirrers, eccentric with baffles). Subsequent levels branch out from the second-level options, providing links to individual frames and their related information.
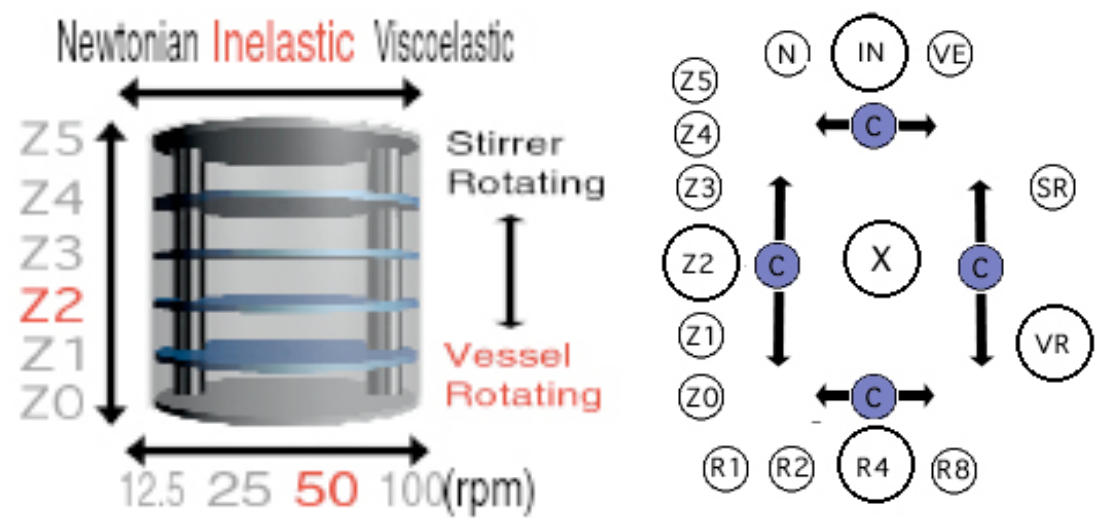

Fig. 5. Multi-menu graph representation for two-stirrer design, contrasted to the user-interface component used under the dough-kneading multimedia system.

Fig. 5, left, shows a section snapshot of organisation for links to MMScomponents. Varying parameters represent settings which trigger the display of content components, animations and sounds. Adjacent settings represent related MM-frames. To represent this hierarchy using a graph, each node must represent a MM-frame. Each such node can inherit attributes from another; new attributes/behaviours may be appended, or replace those previously. This organisation is adaptive and flexible and can be utilised to develop interfaces which may simultaneously employ direct manipulation and adaptation [14]. Attributes and metadata need not be programmed for each frame, being inherited for a copy-object of an existing frame. When an attribute is programmed, it generates a new database 
object that may be associated with any component of a frame. Frame components are stored uniquely within the database and are referenced to the same item, when multiple instances are on display. Instead, a link is employed, if many instances of the same data element are required. The user may select when the memory is flushed of that particular element.

Up to this point, user-driven investigation was implemented through the use of visual database queries. Parameter selection triggered a series of events where with the use of metadata encapsulated under each frame, appropriate streams were selected, pre-cached, positioned and ultimately displayed. Dynamic and adaptive linking may be implemented via Multi-Menus, even with primitive programming tools provided under propriety interactive multimedia environments. This involves the specification of an external mechanism (for example a knowledge-base), which SIMM accesses when multiple-links are encountered. The full complexity of such external decision-making may be implemented within a MME. One method to integrate decision-making procedures, within the MME, is through the use of global data-structures. These may be tables or linked-lists and may be employed to store information at runtime. Multi-Menus may access these global structures directly, by reference to structure-name.

\section{Conclusions}

Multi-Menus are an interaction structure that beyond the support for advanced, automated and user-defined interaction, offers the ability to present results comparatively, from case to case, or via interactive mode-switching, a highly desirable presentation characteristic. Research in this interdisciplinary area of interactive multimedia has resulted in the development of a wide range of MMS, destined for courseware, research and industrial applications [3]. We have shown that combined use of the framework, model, metadata-based multi-menus and internal or external rule-based reasoning may be used as an effective method to create useful research and presentation tools for customised fields of research, where propriety presentation software has been found lacking. The system thus developed has been used actively to cover wide-ranging presentation requirements, addressing, in particular, industrial and academic audiences. Also, it displays the potential to reach more general/school audiences. The choice of a fully programmable Multimediadevelopment platform for the Internet (Adobe Director) prevented presentation deficiencies and limitations introduced with proprietary software, enabling webdelivery to be implemented without the need for additional end-system reprogramming. 


\section{References}

1. Botafogo, R.A. and D. Mossé, The Capoeira Model for Distributed and Reconfigurable Multimedia Systems. 1996, Computer Science Department, Universidade Federal Fluminense: Brazil.

2. Carter, J. Developments in Web Research and Practice. in Motivating Multimedia. 2000. San Diego, California, US.

3. Deliyannis, I., Interactive Multimedia Systems for Science and Rheology, in Computer Science. 2002, Ph.D Thesis, University of Wales: Swansea. p. 285.

4. Deliyannis, I. and M.F. Webster, A Multimedia Investigation Environment for Rheological Contraction-Flow data-sets. The Mathematical Review, Hellenic Mathematical Society, 2006(64).

5. Deliyannis, I. and M.F. Webster. Multi-Level Modelling and Interactive Multimedia presentation of Scientific Data over various Media. in IADIS. 2002. Lisbon Portugal.

6. Deliyannis, I. and M.F. Webster. WWW Delivery of Graph-Based, Multi-Level Multimedia Systems: Interaction over Scientific, Industrial and Educational Data. in IADIS Int. Conf. WWW/Internet 2002. 2002. Lisbon Portugal.

7. Doherty, G. and M.D. Harrison. A Representational Approach to the Specification of Presentations. in $4^{\text {th }}$ Eurographics Workshop on the Design Specification and Verification of Interactive Systems (DSV-IS '97). 1997. Granada, Spain.

8. Geurts, J.P.T.M., J.R.v. Ossenbruggen, and H.L. Hardman. Application-specific constraints for multimedia presentation generation. in Eighth International Conference on Multimedia Modeling (MMM01). 2001. Amsterdam, Netherlands.

9. ISO-Standard, Information technology - Computer graphics and image processing Presentation Environment for Multimedia Objects (PREMO). 1988.

10. J. Dakss, et al. Hyperlinked Video. in Proc. SPIE Multimedia Systems and Applications. 1998.

11. Martinez, O.R., Design Dependencies within the Automatic Generation of Hypermedia Presentations. 2002, Technical University of Catalonia: Catalonia.

12. Pittarello, F. and A. Celentano. Interaction locus: a multimodal approach for the structuring of virtual spaces. in HCItaly. 2001. Firenze, Italy.

13. Rees, D.L.L., K. Stephenson, and J.V. Tucker, Towards an Algebraic Structure of Interface Definition Languages and Architectures. 2000, Computer Science Department, University of Wales Swansea: Swansea.

14. Tsandilas, T. and M.C. Schraefel, Usable adaptive hypermedia systems. New Review of Hypermedia and Multimedia, 2004. 10(1): p. 5-29.

15. Vazirigiannis, M., Y. Theodoridis, and T. Sellis, Spatio-temporal composition and indexing for large multimedia applications. Multimedia Systems, 1998. 6: p. 284298.

16. Wilson, M.D., Enhancing Multimedia Interfaces with Intelligence. Multimedia Systems and Applications, 1995. 